\title{
The Effect of Anaesthesia on Oral Fibreoptic Tracheal Intubation in A Patient of Ankylosing Spondylitis
}

\author{
Weiqian Tian* \\ Department of Anaesthesiology, Affiliated Hospital of Nanjing University of Chinese Medicine, China
}

*Corresponding author: Weiqian Tian, Department of Anaesthesiology, Affiliated Hospital of Nanjing University of Chinese Medicine, Nanjing, Jiangsu Province, China.
Received Date: June 10, 2020

Published Date: June 30, 2020

\begin{abstract}
Airway management in patients with ankylosing spondylitis (AS) is a challenging problem for anaesthesiologists. The fibreoptic intubation (FOI) is designed to assist tracheal intubation for patients with a difficult airway. If we chose to do FOI, our first step is to decide whether to do a fibreoptic intubation with the patient anesthetized or awake. Once we've decided on anesthetized or awake, chose either the oral or nasal route. The aim of the study was to report the successful intubation by anesthetized nasal fibreoptic tracheal intubation of an AS patient after the failed intubation by anesthetized oral fibreoptic tracheal intubation because of a difficult airway found, and to discuss the effect of anaesthesia on oral fibreoptic tracheal intubation in patients with AS. One patient with chronic, severe AS were evaluated preoperatively and had features associated with a difficult direct laryngoscopy. Awake oral fibreoptic intubation was recommended to the patient. Patients were kept in supine position, with their head and neck supported on pillows. We performed the necessary preparations for difficult airway and intubation. First, we attempted awake fibreoptic orotracheal intubation. When a gap was observed between the epiglottis and posterior pharyngeal wall and wanted to further advance the instrument into the gap, Patient was unable to cooperate and presented with irritable cough. So, we decided to perform anesthetized oral fibreoptic tracheal intubation. Following sufficient preoxygenation, patients received i.v. sufentanil at $0.4 \mu \mathrm{g} / \mathrm{kg}$, propofol at $2 \mathrm{mg} / \mathrm{kg}$, and rocuronium at $1 \mathrm{mg} /$ kg. Ninety seconds after the rocuronium administration, Fibreoptic bronchoscopy was attempted again. However, although anaesthesia provided skeletal muscle relaxation, but oropharyngeal and laryngeal muscle relaxation resulted in upper airway collapse. The upper airway collapse made fibreoptic visualization of the glottis difficult. After multiple attempts with oral fibreoptic laryngoscopy failed, nasal fibreoptic intubation was then performed with the jaw thrust manoeuvre. Fibreoptic laryngoscopy confirmed the glottis rapidly. Intubation was accomplished successfully with a 6.5 endotracheal tube lubricated with lidocaine gel. Surgery proceeded uneventfully, and the postoperative course was uncomplicated. Awake oral fibreoptic intubation was ideal and safe to secure airway in severe AS patients, but an anesthetized oral fibreoptic tracheal intubation could be difficult to do that. Anesthesia decreased muscle tone in upper airway, especially at the level of the soft palate and the epiglottis. As the depth of general anesthesia increases, upper airway narrowing occurs throughout the entire upper airway but is most pronounced in the hypopharynx at the level of the epiglottis. Thus, making fibreoptic visualization of the glottis difficult. Anesthesia also prevented the patient from inspiring deeply, which could help locate the laryngeal inlet. In this situation, we might have to resort to nasal fibreoptic tracheal intubation. So do not do oral fibreoptic intubations on anesthetized patients with AS. Nasal fibreoptic intubation was more successful and easier than the oral approach in anesthetized patients with AS.
\end{abstract}

\section{Introduction}

Ankylosing spondylitis is a painful, chronic inflammatory arthropathy, which primarily affects the spine and sacroiliac joints and eventually leads to fusion and rigidity of the spine (bamboo spine) [1]. Awake fibreoptic intubation (FOI) is the gold standard technique for airway management of such patients with a stiff spine [2]. Moreover, orotracheal tubes are preferred over the nasotracheal tubes as the latter are associated with greater chances of sinusitis and nasal trauma [3]. Awake oral fibreoptic intubation is ideal and safe to secure airway in these patients because of no need of neck mobilization. However, it is an uncomfortable procedure, and thus many patients may refuse and resist the technique. To minimize movement during intubation and attain appropriate position was of prime concern, fibreoptic intubation under anaesthesia may be chosen in patients who were otherwise neither able to cooperative 
nor able to give appropriate position for FOI. However, anaesthesia could result in respiratory depression and affect the upper airway patency, making FOI difficult and sometimes impossible. We hereby report airway management of a ankylosing spondilytic patient who suffered from the failed intubation by anesthetized oral fibreoptic tracheal intubation.

\section{Case Report}

A 46-years-old man (height $160 \mathrm{~cm}$; weight $60 \mathrm{~kg}$ ) with severe AS, as well as a history of AS for 20 years, was admitted to our hospital's urology department for distal ureteral stone. An assessment of the airway showed a mouth opening of $3 \mathrm{~cm}$, a thyromental distance of $5.5 \mathrm{~cm}$, grade III Mallampati, and restricted neck mobility with fixed and rigid flexion and extension. Other investigations like hemogram, liver function test, renal function test and electrocardiogram were unremarkable. As spinal anaesthesia might not be performed successfully, general anaesthesia with tracheal intubation was required for the surgery. Preparations were made for difficult intubation and the anaesthesia procedure for AS was applied to the patient. Awake fibreoptic intubation was recommended to the patient. Patient was explained about awake FOI.

In operation room, all standard monitors were attached. Patient was asked to position himself in a comfortable posture. Two pillows were kept under head to accommodate acute curvilinear posture of the spine. Patient was nebulized with $2 \%$ lidocaine for $10 \mathrm{~min}$ and 5 $\mathrm{mL}$ of $2 \%$ lidocaine was injected through thoracentesis. Patient was started on dexmedetomidine infusion, with $1 \mu \mathrm{g} / \mathrm{kg}$ induction dose over $10 \mathrm{~min}$ followed by infusion of $0.5 \mu \mathrm{g} / \mathrm{kg}$. Supplemental oxygen was administered through nasal cannula. Bite block was placed, and fibreoptic bronchoscopy was attempted in the same position. First we attempted awake oral fibreoptic tracheal intubation in a neutral position. When a gap was observed between the epiglottis and posterior pharyngeal wall and wanted to further advance the instrument into the gap, patient was unable to cooperate and presented with irritable cough. So we decided to perform oral fibreoptic tracheal intubation under anaesthesia.

Following sufficient preoxygenation, patients received i.v. remifentanil at $1 \mathrm{lg} \mathrm{kg}-1$, propofol at $2 \mathrm{mg} \mathrm{kg}-1$, and rocuronium $(1 \mathrm{mg} / \mathrm{kg})$. Ninety seconds after the rocuronium administration, Fibreoptic bronchoscopy was attempted again. However, although anaesthesia provided skeletal muscle relaxation, but oropharyngeal and laryngeal muscle relaxation resulted in airway collapse. Despite multiple attempts at oral fibreoptic laryngoscopy using the Ovassapian Fibreoptic Intubating Airway and applying a chin lift/jaw thrust, neither the epiglottis nor the vocal cords could be identified. After multiple attempts with oral fibreoptic laryngoscopy failed, nasal fibreoptic intubation was then performed with the jaw thrust manoeuvre.

Fibreoptic laryngoscopy confirmed the glottis. The epiglottis was noted to be deviated to the left of the midline, and the vocal cords could be visualized. Intubation was accomplished successfully with a 6.5 endotracheal tube lubricated with lidocaine gel. Surgery proceeded uneventfully, and the postoperative course was uncomplicated.

\section{Discussion}

Ankylosing spondylitis is a progressive, seronegative, inflammatory rheumatic arthropathy affecting the bony insertion points of ligaments. The spine and sacroiliacjoints are predominantly affected. Over time, the vertebral column ossifies and results in a progressive loss of spinal mobility and a characteristic "bamboo spine" on imaging, which depicts the bridging bone [4]. Deformity and stiffness lead to decreased functionality and progressive osteoporosis. The spine is thus rendered more susceptible to fracture from smaller physical loads, making standard intubation techniques difficult and sometimes impossible.

To improve ease and success, and reduce cervical spine movement, awake fibreoptic intubation (FOI) is considered the gold standard technique for airway management in such cases.

As this patient had a difficult airway and was likely to be ventilated in the postoperative period, we preferred oral route. Orotracheal tubes are preferred over the nasotracheal tubes as the latter are associated with greater chances of sinusitis and nasal trauma often follows with nasal intubation. Our routine protocol is to use orotracheal route.

In our case, the patient was supported with pillows for comfort, airway blocks were done with great difficulty due to distorted anatomy and difficult access. We decided to do awake oral FOI, but it was very uncomfortable for the patient and difficult for the performer. For awake FOI, patient comfort and optimal intubating conditions both are of paramount importance. Attaining appropriate position for intubation was challenge in this case due to rigid curvature of the ankylosed spinal column. Then the patient was started on dexmedetomidine infusion to provide sedation and analgesia. Patient was unable to cooperate and presented with irritable cough when we saw the view of the glottis at fibreoptic bronchoscopy. So, we decided to perform oral fibreoptic tracheal intubation under anaesthesia. However, anaesthesia let us suffer from an unexpected failed FOI as the view of the glottis could not be seen again. Anaesthesia led to the upper airway collapse that made oral FOI very difficult. The upper airway collapse in the anesthetized patient is the result of loss of tonicity of the submandibular muscles, which provide direct support of the tongue and indirect support to the epiglottis [5]. As a result of this loss of tonicity, posterior displacement of the tongue may occlude the airway at the level of the pharynx, and the epiglottis may occlude the airway at the level of the larynx. The pharyngeal airway space decreases with deepening anaesthesia. This reduction in pharyngeal space has been observed mainly in the anteroposterior dimension. As the depth of general anaesthesia increases, upper airway narrowing occurs throughout the entire upper airway but is most pronounced in the hypopharynx 
at the level of the epiglottis [6]. Thus, making fibreoptic visualization of the larynx and placement of the endotracheal tube difficult. Deep anaesthesia also prevented the patient from inspiring deeply, which could help locate the laryngeal inlet. So do not do oral fibreoptic intubations on anesthetized patients in whom a severe difficult airway has been expected.

If the anesthetized oral approach proves unsuccessful because of a difficult airway found on oral fibreoptic laryngoscopy, you may need to resort to an anesthetized nasal approach. Because nasal fibreoptic intubation is more successful and easier than the oral approach in anesthetized patients.

\section{Acknowledgement}

None.

\section{Conflict of Interest}

The authors declare no competing interest.

\section{References}

1. Rakhee G, Shivinder S, Ravindra Nath S, Anuj S (2013) Management of a case of ankylosing spondylitis for total hip replacement surgery with the use of ultrasound-assisted central neuraxial blockade. Indian J Anaesth 57(1): 69-71
2. Nilesh K, Ashish B, Charu M, Naveen Y (2015) Airway management in a patient of ankylosing spondylitis with traumatic cervical spine injury. Saudi J Anaesth 9(3): 327-329.

3. Lili Plümer, Gerhard Schön, Jan Klatt, Henning Hanken, Rainer Schmelzle, et al. (2014) Nasal position of nasotracheal tubes: a retrospective analysis of intraoperatively generated three-dimensional X-rays during maxillofacial surgery. Eur J Med Res 19(1): 55.

4. Ul Haq MI, Shamim F, Lal S, Shafiq F (2015) Airway Management in a Patient with Severe Ankylosing Spondylitis Causing Bamboo Spine: Use of Aintree Intubation Catheter. J Coll Physicians Surg Pak 25(12): 900902.

5. De Jarnett D (2013) Use of a positive pressure endoscopic mask to assist with positive pressure ventilation in a morbidly obese patient during fiberoptic intubation: a case report. AANA J 81(4): 282-284.

6. Machata AM, Kabon B, Willschke H, Prayer D, Marhofer P (2010) Upper airway size and configuration during propofol-based sedation for magnetic resonance imaging: an analysis of 138 infants and children. Paediatr Anaesth 20(11): 994-1000. 